\title{
Vantaggi e limiti delle tecniche emodialitiche
}

\author{
A. Santoro, M. Spongano \\ Divisione di Nefrologia e Dialisi, Ospedale Malpighi, Policlinico S. Orsola-Malpighi \\ Bologna
}

1 rene come organo svolge la sua funzione di controllore del mezzo interno attraverso una serie di complessi meccanismi che richiedono: forze idrauliche, barriere selettive di permeabilità, sistemi di trasporto specializzati e precisi controlli a retroazione. La sostituzione di una funzione così articolata e polivalente si scontra nella pratica con i limiti della conoscenza e la disponibilità delle tecnologie.

L'apparente elegante semplicità dell'emodialisi, con un banale circuito idraulico, una pompa ed un filtro è solo il frutto della nostra incapacità nell'imitare un organo strutturalmente e funzionalmente complesso. Eppure il trattamento sostitutivo della funzione renale è ormai entrato nella quinta decade della sua applicazione clinica ed il numero di pazienti che vivono grazie ad esso si avvia a raggiungere il milione di individui. Tuttavia, dalle prime esperienze di Kolff (1) ai nostri giorni, molti aspetti e finalità sono profondamente cambiati. Negli anni della sua nascita il rene artificiale veniva visto come una misura salva vita. L'obiettivo principale della sua applicazione clinica, dopo la sopravvivenza del paziente, era il controllo delle gravi complicanze (l'ipertensione, la pericardite, l'encefalopatia), che potevano risultare fatali per il paziente con insufficienza renale. Passati venti anni dalla sua applicazione clinica, e quindi già negli anni ' 80 , data per scontata la sopravvivenza, il vero obiettivo da perseguire diventava la prevenzione delle complicanze a lungo termine come l'artropatia, l'amiloidosi, la cardiomiopatia.

Ai nostri giorni i criteri oggettivi e le modalità di realizzazione di una terapia adeguata e soprattutto individualmente adeguata sono i traguardi verso cui stiamo andando.

In realtà ancora poco sappiamo sui complessi meccanismi fisiopatologici e sulle interrelazioni che intervengono tra i vari fattori patogenetici che generano la sindrome uremica. Il tempo e l'esperienza ci hanno insegnato che è possibile mantenere in vita un paziente che abbia perso la funzione dei propri reni attraverso un controllo anche approssimativo del bilancio dei liquidi e dell'equilibrio acido-base, nonché con la rimozione, sia pure incompleta, di una serie di prodotti "tossici" del metabolismo intermedio.

Da questa visione estremamente pratica e semplicistica della sostituzione della funzione renale restano esclusi ed ignorati quei complessi e sofisticati meccanismi che solo il rene umano è in grado di realizzare. Sul piano pratico la nostra incapacità conoscitiva, accanto alla artificiosità stessa delle tecnologie applicative, può non solo portarci a delle imita- zioni estremamente incomplete ma anche dar luogo a patologie iatrogeniche, come la stessa amiloidosi, che si inseriscono nel complesso e proteiforme quadro della sindrome uremica.

Pertanto le spinte verso l'evoluzione della tecnica dialitica possono derivare: a) dal miglioramento delle nostre conoscenze sugli aspetti funzionali del rene; b) dal progresso tecnico-scientifico; c) dal nostro desiderio di non indurre effetti collaterali con l'applicazione della terapia.

Sul piano strettamente clinico gli obiettivi fondamentali di un trattamento dialitico ottimale restano:

- una lunga sopravvivenza ( $\geq 20-30$ anni);

- una bassa morbidità;

- la prevenzione delle complicanze cardiovascolari, osteoarticolari, neurologiche, nutrizionali ed ormonali;

- la riabilitazione del paziente sul piano psicologico e socio-professionale. La realizzazione di questi obiettivi deve però confrontarsi con le caratteristiche della popolazione dialitica e gli ultimi dieci anni hanno visto un incremento progressivo della criticità clinica dei pazienti in dialisi. L'età media si è spostata verso la sesta, settima decade e consensualmente vi è stato un incremento delle patologie sistemiche (diabete, cardiovasculopatie, ecc.) di accompagnamento. 
La tecnologia si è dovuta adeguare ed evolvere sulla base delle modifiche delle caratteristiche della popolazione da trattare e delle nuove aspirazioni dei nefrologi.

\section{Evoluzione della tecnologia dialitica}

A parte la CAPD che rappresenta dal punto di vista concettuale e pratico una reale e concreta alternativa, le nuove varianti tecniche dell'emodialisi convenzionale, compresa l'emofiltrazione, non sono che emanazioni di quanto prospettato da Kolff nel lontano 1946 (1). L'emodialisi tradizionale basata sul cuprofan e sull'impiego dell'acetato ha rappresentato, sino ad alcuni anni fa, il trattamento sostitutivo standard ed ottimale. Ancora nel 1989 l'acetato dialisi era la tecnica più diffusa e più applicata in Europa (2) e solo nel 1990 veniva superata dalla dialisi con bicarbonato (3). Le motivazioni che, accanto al progresso tecnologico, hanno favorito lo sviluppo di tecniche alternative sono diverse e talora in contrasto tra loro:

- correzione ottimale dell'acidosi uremica;

- rimozione di un ampio range di soluti (dalle piccole alle grandi molecole); - rimozione di elevate quantità di piccole molecole;

- riduzione dei tempi di trattamento;

- buona stabilità emodinamica intradialitica;

- biocompatibilità del circuito extracorporeo.

Alcune di queste aspettative si sono concretizzate grazie allo sviluppo tecnologico che ha accompagnato il progresso conoscitivo permettendo di realizzare delle tappe importanti nella storia del rene artificiale.

L'arrivo di membrane ad alta permeabilità idraulica ed elevato "cut-off" ha permesso lo sviluppo di trattamenti basati sulla convezione e di tecniche miste convettivo-diffusive. $\mathrm{Ne}$ è derivato un $\mathrm{mi}$ glioramento nella rimozione di soluti sia in termini di piccole che di medie molecole.

L'aumento dell'efficienza sul piano depurativo ha alimentato e permesso una riduzione dei tempi di trattamento.

L'accorciamento dei tempi di dialisi ha però smascherato, esaltandoli, tutti i di- fetti del tampone acetato sia sul piano metabolico che su quello cardiovascolare. Il bicarbonato è diventato il tampone d'obbligo nei trattamenti brevi e ad alta efficienza. Nonostante però l'utilizzo del bicarbonato, trattamenti diffusivi troppo brevi e troppo efficienti sono in grado di indurre sindromi da squilibrio osmotico ed emodinamico (4). Al contrario transfer convettivi, anche se efficienti e rapidi, sono molto meno destabilizzanti nei confronti del sistema cardiovascolare di quelli diffusivi (5). L'emodiafiltrazione e, soprattutto, l'emofiltrazione sono in grado di assicurare una migliore stabilità emodinamica di quanto non facciano le classiche tecniche diffusive. Inoltre le tecniche convettive che presuppongono l'impiego di membrane sintetiche facilitano la rimozione di soluti di elevato peso molecolare.

Negli ultimi anni la tossicità di medie e grandi molecole, come la Beta2-microglobulina, è stata evidenziata e correlata ad alcuni aspetti della patologia del dializzato.

Un accumulo iatrogenico, connesso all'impiego di materiali e membrane non perfettamente biocompatibili, può però essere ipotizzato per molte delle molecole tossiche dell'uremia. L'attivazione di alcune proteine plasmatiche della cascata enzimatica della coagulazione e del complemento possono innescare una serie di eventi metabolici che va sotto il nome di "acute phase reaction", respon- sabile della produzione ed attivazione di molecole ad elevata attività biologica (6). La sostituzione di membrane cellulosiche con membrane sintetiche ad alta permeabilità idraulica permette, da un lato, di ridurre la bioincompatibilità della membrana e, dall'altro, di sfruttarne le caratteristiche strutturali nella rimozione tanto di piccole che di medie molecole.

L'elevata permeabilità idraulica delle membrane non cellulosiche accanto ad una minore funzione di setacciamento espone però ad un maggior rischio di back-filtration (7). All'estremità venosa del dializzatore, dove il gradiente di pressione tra sangue e liquido di dialisi è a favore di quest'ultimo, può aversi il passaggio nel compartimento ematico, insieme con i flussi acquosi, di contaminanti del liquido di dialisi. Particolarmente dannoso può risultare il trasferimento di frazioni endotossiniche risultanti dalla contaminazione batterica dei concentrati. Evenienza questa non infrequente nei concentrati liquidi basici che, privi di qualsiasi attività batteriostatica, possono favorire la proliferazione batterica ( 8 )

Il semplice contatto delle cellule mononucleate del sangue con endotossine o frazioni di esse è in grado di innescare la rapida sintesi di mRNA per l'Interleukina-1 ed il TNF, dando luogo ad una vera e propria cascata biologica che può comportare la liberazione ed il successivo accumulo di una serie di mediatori

TAB. I - TECNICHE DIALITICHE E RISPETTIVE MEMBRANE UTILIZZABILI
Tecnica
Membrane
Emodialisi convenzionale
Cellulosiche, sintetiche a bassa permeabilità
Emodialisi ad alta efficienza
Cellulosiche, sintetiche a bassa permeabilità, ampia superficie

Emodialisi high-flux

Sintetiche, alta permeabilità

Emofiltrazione

Sintetiche, alta permeabilità

Emodiafiltrazione

Sintetiche, alta permeabilità

Acetate Free Biofiltration

Sintetica (PAN), alta permeabilità

Paired Filtration Dialysis
Sintetica ad alta permeabilità (convezione) cellulosica o sintetica a bassa permeabilità (diffusione) 


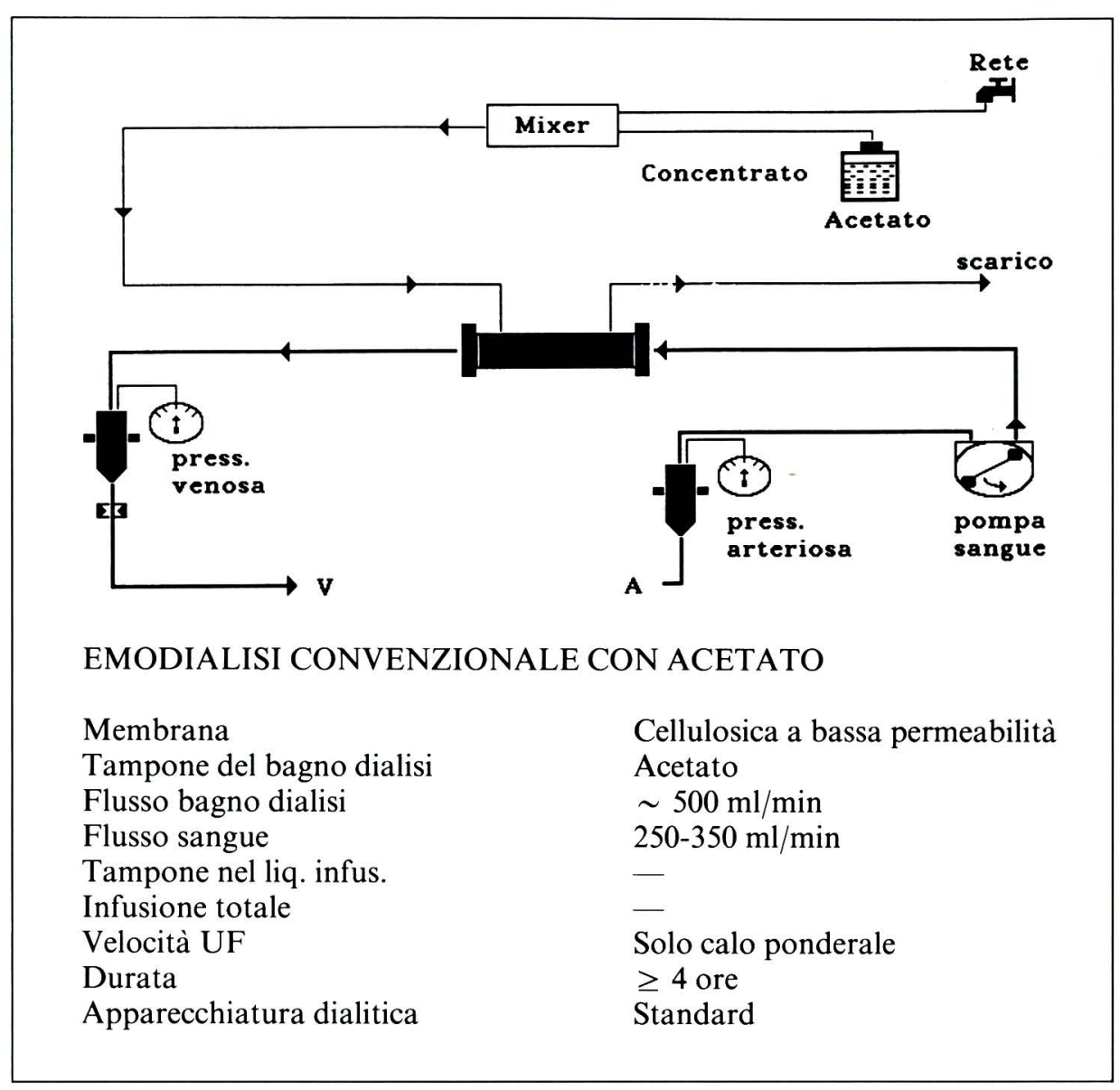

Fig. 1

\section{TAB. II - EMODIALISI CONVENZIONALE CON ACETATO}

\section{Vantaggi:}

- coesistenza dei sali di Calcio e Magnesio con l'acetato nel bagno dialisi

- utilizzo di dializzatori di basso costo

- semplicità e sicurezza delle apparecchiature

- manutenzione e costi di gestione contenuti

\section{Svantaggi:}

- limitato range di rimozione di soluti, con clearance insignificanti per soluti di peso molecolare medio-alto

- insufficiente correzione dell'acidosi, in particolare in pazienti "cattivi" metabolizzatori dell'acetato

effetti emodinamici negativi dell'acetato (riduzione delle resistenze periferiche, incremento della frequenza cardiaca, ipossiemia)

scarsa biocompatibilità delle membrane biologici.

Il rischio potenziale di back-filtration impone con le membrane ad elevato cutoff un controllo continuo ed efficace del bilancio delle pressioni all'interno del dializzatore, l'impiego di dialisato sterile, o il ricorso a tecniche convettive e convettivo-diffusive che siano sempre in grado di assicurare un flusso direzionale dal sangue al liquido di dialisi.

Su queste premesse sono nate in Italia due tecniche dialitiche originali, l'Acetate-Free Biofiltration (AFB) proposta inizialmente da Buoncristiani (9), e la Paired Filtration Dialysis (PFD) concepita e realizzata da Ghezzi (10). Entrambe le tecniche presuppongono l'utilizzo di membrane sintetiche con basso rischio di back-filtration ed in ogni caso con minimizzazione delle conseguenze biologiche di una eventuale retrofiltrazione. Queste tecniche hanno affiancato le numerose differenti strategie e tecnologie che si sono proposte come alternative all'emodialisi convenzionale nell'intento di migliorare la qualità e le prestazioni della terapia emodialitica (Tab. I). Per ognuna delle tecniche elencate possono, a seconda dei differenti punti di vista, essere posti in evidenza vantaggi e svantaggi che ce ne fanno preferire una all'altra. Naturalmente le valutazioni espresse di seguito sono sempre di carattere parziale e frutto di esperienze personali e di confronto con i dati della letteratura.

\section{Emodialisi convenzionale con acetato (Fig. 1)}

Vantaggi (Tab. II)

Resta per il paziente uremico il trattamento standard, in grado di assicurare una lunga aspettativa di vita pur nella sua semplicità. L'emodialisi convenzionale con acetato può essere eseguita facilmente in ospedale, in centri dialisi satelliti, e a domicilio dal paziente stesso. Rappresenta sicuramente la forma più economica di depurazione extracorporea dell'uremico.

Svantaggi (Tab. II)

I suoi svantaggi derivano paradossalmente proprio dai due elementi che la 
TAB. III - EMODIALISI CONVENZIONALE CON BICARBONATO

\section{Vantaggi:}

- buona correzione dell'acidosi

- soddisfacente stabilità cardiovascolare intradialitica

\section{Svantaggi:}

- limitato range di rimozione di soluti

- scarsa biocompatibilità delle membrane

- rischio di contaminazione batterica ed endotossinica dei concentrati basici

- possibilità di errori nella miscelazione dei concentrati acidi e basici

- complessità delle apparecchiature che richiedono un sistema di preparazione del bagno dialisi supplementare

- necessità di una accurata e frequente manutenzione delle apparecchiature; continue procedure di lavaggio del circuito idraulico con soluzioni decalcificanti

- necessità di concentrati a differenti concentrazioni di bicarbonati per poter variare l'apporto di basi al paziente

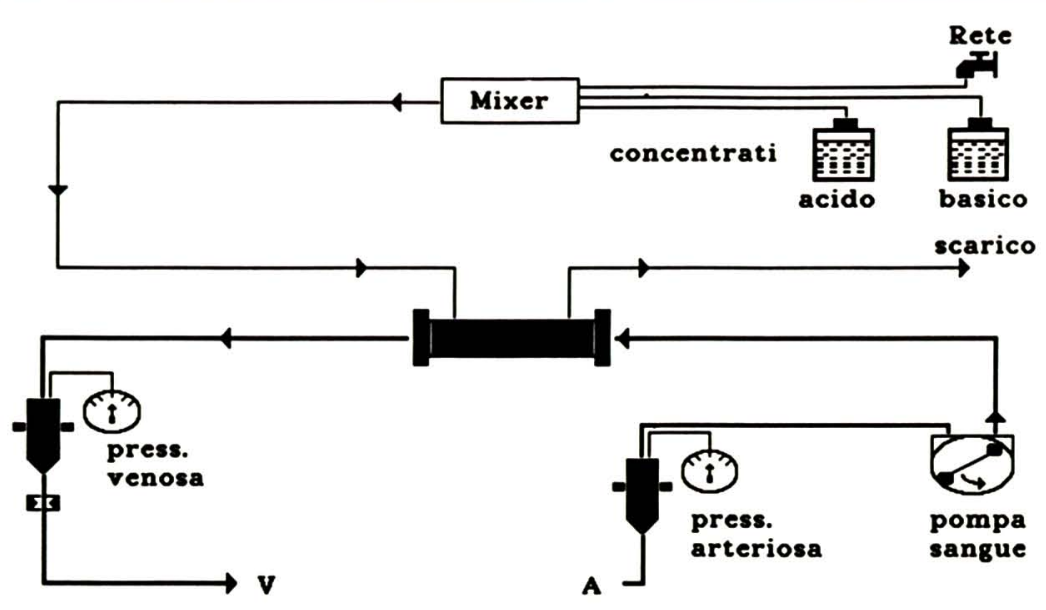

\section{EMODIALISI CONVENZIONALE CON BICARBONATO}

\section{Membrana}

Tampone del bagno dialisi

Flusso bagno dialisi

Flusso sangue

Tampone nel liq. infus.

Infusione totale

Velocità UF

Durata

Apparecchiatura dialitica
Cellulosica a bassa permeabilità

Bicarbonato

$\sim 500 \mathrm{ml} / \mathrm{min}$

$250-350 \mathrm{ml} / \mathrm{min}$

-

Solo calo ponderale

$\geq 4$ ore

Circuito idraulico addizionale per la miscelazione dei 2 concentrati

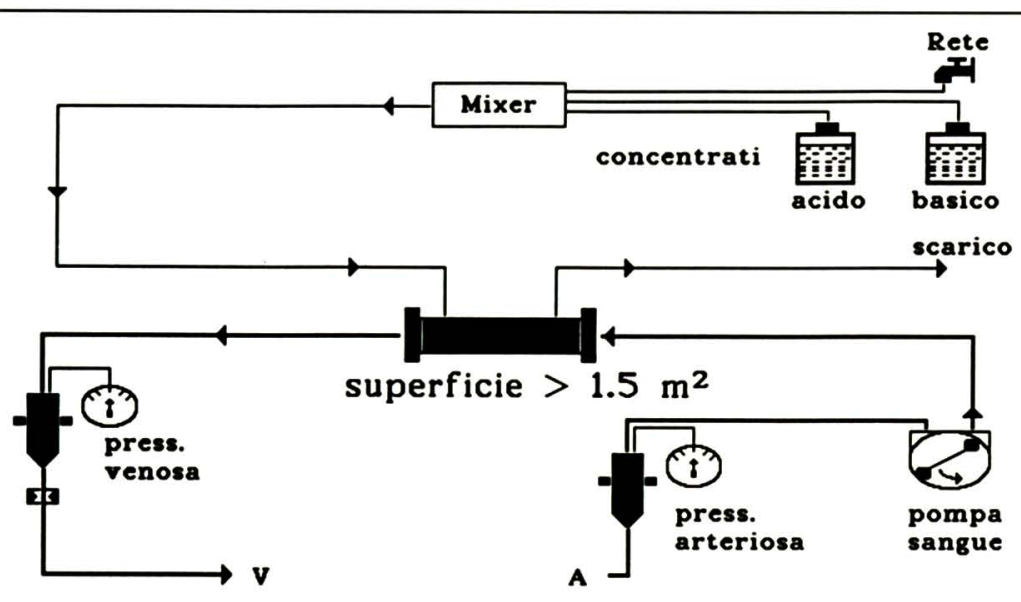

\section{EMODIALISI AD ALTA EFFICIENZA}

\section{Membrana}

Tampone del bagno dialisi

Flusso bagno dialisi

Flusso ematico

Tampone del liq. infus.

Infusione totale

Velocità UF

Durata

Apparecchiatura dialitica
Cellulosica ad ampia superficie $\left(>1.5 \mathrm{~m}^{2}\right)$

Bicarbonato

$\sim 500 \mathrm{ml} / \mathrm{min}$

$\geq 300 \mathrm{ml} / \mathrm{min}$

-

Solo calo ponderale

$<4$ ore

Standard
TAB. IV - EMODIALISI AD ALTA EFFICIENZA

\section{Vantaggi:}

- possibilità di riduzione dei tempi di trattamento in rapporto all'elevata efficienza

\section{Svantaggi:}

- maggiori rischi di sindromi da squilibrio osmotico

- stessi svantaggi derivanti dall'uso di membrane cellulosiche (bioincompatibilità, aumento dei trasnfer solo per soluti di basso peso molecolare)

- problemi tecnici legati all'uso del bicarbonato

- bilanci sodici non sufficientemente negativi con concentrazioni sodiche elevate nel dializzato 
rendono semplice e vantaggiosa: le membrane cellulosiche e l'acetato. La bassa clearance dei soluti di elevato peso molecolare, derivante dall'impiego delle membrane cellulosiche, l'inadeguatezza nella correzione dell'acidosi e, soprattutto, i side-effects vascolari, la cosiddetta "intolleranza all'acetato", sono comunemente ritenuti i suoi principali inconvenienti.

\section{Emodialisi convenzionale con bicarbonato (Fig. 2)}

Vantaggi (Tab. III)

Sul piano metabolico la sostituzione dell'acetato con bicarbonato ci riporta ad una più fisiologica correzione dell'acidosi uremica con indubbi vantaggi anche sulla stabilità cardiovascolare intradialitica.

\section{Svantaggi (Tab. III)}

La necessità di un circuito idraulico addizionale per la preparazione del bicarbonato rende più complesse le attrezzature aumentandone i costi. Il rischio di precipitazione di sali insolubili di calcio accanto all'eventualità di errori nella selezione dei concentrati, e sempre e comunque presente. La facile proliferazione batterica nelle soluzioni basiche soprattutto in condizioni precarie di stoccaggio (ambienti con alte temperature e poco areati) espone ad un continuo rischio di contaminazione del circuito del dializzante.

\section{Emodialisi ad alta efficienza (Fig. 3)}

\section{Vantaggi (Tab. IV)}

Gli elementi essenziali all'emodialisi tradizionale ad alta efficienza sono l'impiego di membrane cellulosiche (in genere superiori a $1.5 \mathrm{~m}^{2}$ di superficie) in grado di assicurare elevate clearance dell'urea ad alti flussi sangue e con basse velocità di ultrafiltrazione (inferiori ai 10 $\mathrm{ml} / \mathrm{h} / \mathrm{mmHg}$ ), il tampone bicarbonato, linee ematiche ed aghi in grado di sopportare alte pressioni e flussi superiori ai

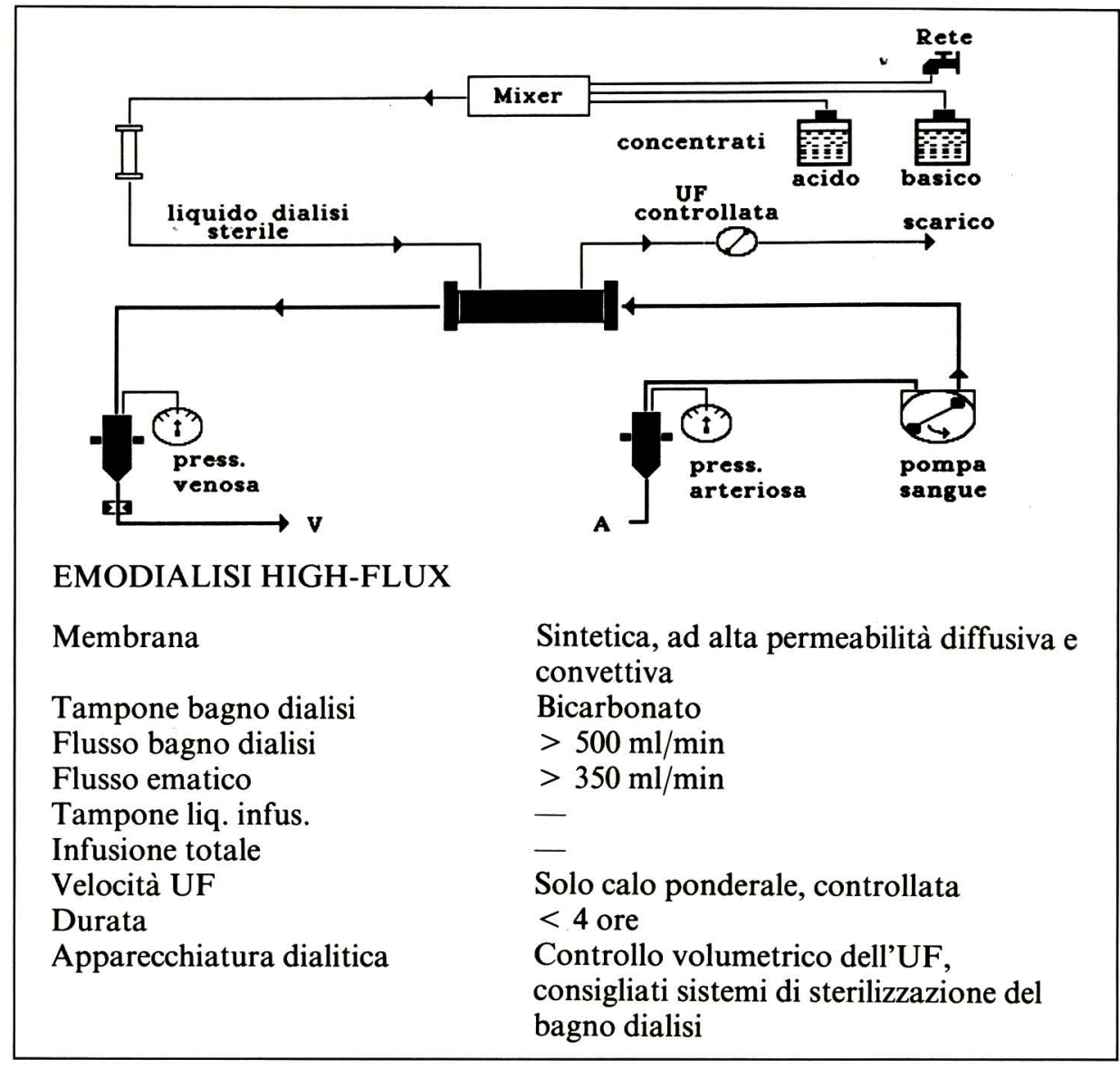

$350-400 \mathrm{ml} / \mathrm{min}$.

Fig. 4

Un'adeguata rimozione di soluti di piccolo peso molecolare può essere facilmente ottenuta anche con tempi di trattamento relativamenti brevi $(\leqslant 180$ $\min )$.

Svantaggi (Tab. IV)

L'impiego di membrane cellulosiche comporta sia sul piano della biocompatibilità che su quello della rimozione dei soluti gli stessi limiti dell'emodialisi convenzionale. Nel paziente critico l'accorciamento dei tempi di trattamento mal si concilia con la tolleranza dialitica e può facilitare la comparsa di sindromi da squilibrio osmotico.

\section{Emodialisi high-flux (Fig. 4)}

\section{Vantaggi (Tab. V)}

La sostituzione del cuprofan e dei suoi derivati con membrane sintetiche risulta
TAB. V - EMODIALISI HIGH-FLUX

Vantaggi:

- ampio range di rimozione dei soluti

- membrane biocompatibili

- possibilità di riduzione dei tempi di trattamento

Svantaggi:

- rischio di back-filtration

- maggiore complessità delle apparecchiature, che devono essere dotate di un efficace controllo volumetrico dell'ultrafiltrazione

- necessità di flussi ematici elevati, non sempre ottenibili 
vantaggiosa in termini di biocompatibilità e clearance di molecole di medio-alto peso molecolare come la Beta2-microglobulina (11400 Daltons).

\section{Svantaggi (Tab. V)}

L'elevata permeabilità idraulica obbliga all'uso di complessi sistemi per il controllo volumetrico dell'ultrafiltrazione. In rapporto a modifiche delle pressioni di ingresso e di uscita dal dializzatore e della pressione oncotica, possono aversi delle inattese e non quantificabili retrofiltrazioni del dialisato.

In presenza di dialisato non sterile possono aversi severe reazioni pirogeniche derivanti dalla produzione ed accumulo di interleukina e altre citokine.

\section{Emofiltrazione (Fig. 5)}

Vantaggi (Tab. VI)

L'uso di membrane sintetiche ad elevata velocità di ultrafiltrazione permette la rimozione dei soluti per convezione sulla base del sieving coefficient della

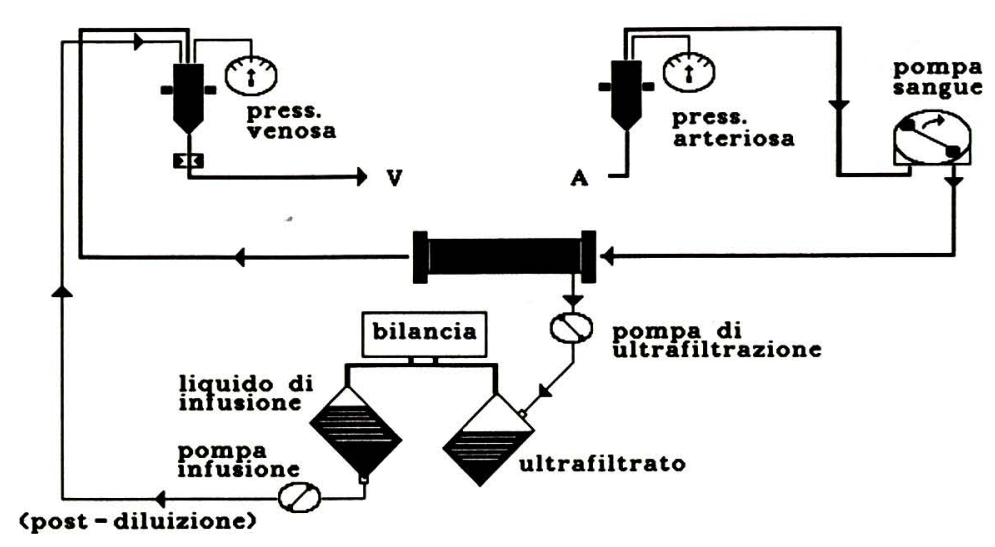

EMOFILTRAZIONE

Membrana

Flusso ematico

Tampone liq. infus.

Infusione totale

Velocità UF

Durata

Apparecchiatura dialitica
Sintetica ad alta permeabilità convettiva $>350 \mathrm{ml} / \mathrm{min}$

Lattato

$20-40 \mathrm{~L}$ in post-diluizione $>40 \mathrm{~L}$ in prediluizione

In base a $\mathrm{Qb}, \mathrm{Hmt}$, membrana

In base a infusione totale, pre- o postdiluizione, membrana

Bilancio di UF e infusione di tipo gravimetrico, sistema di riscaldamento dell'infusione

TAB. VI - EMOFILTRAZIONE

\section{Vantaggi:}

- membrane biocompatibili

- migliore stabilità cardiovascolare intradialitica rispetto alle tecniche diffusive

- migliore controllo dell'ipertensione arteriosa volume-dipendente per una maggiore facilità di raggiungimento del peso secco

- migliore rimozione di medie e grandi molecole rispetto alle altre tecniche (ad esempio Beta2-microglobuline)

- nessun rischio di back-filtration

Svantaggi:

- scarsa rimozione di soluti di basso peso molecolare

- necessità di flussi ematici elevati non ottenibili in caso di accessi vascolari critici

- scarsa correzione dell'acidosi in pazienti che non metabolizzano sufficientemente il lattato

- alti costi (apparecchiature, soluzioni di reinfusione)

- maggiore complessità delle apparecchiature dialitiche

- ingenti quantità di liquido di sostituzione e quindi maggiore...

- ...rischio di contaminazione chimica ( $\mathrm{Al}$, nitrati, cloramine, elementi traccia) e biologica delle soluzioni infusionali

membrana stessa. Biocompatibiltà, assenza di retrofiltrazione, rimozione di molecole di media e grossa taglia, sono gli elementi di spicco di questa tecnica. Una migliore stabilità vascolare ed un minore stress dialitico sono stati documentati rispetto alle metodiche dialitiche diffusive.

\section{Svantaggi (Tab. VI)}

Costi a parte, una ridotta clearance delle piccole molecole a bassi volumi di liquido scambiato e la necessità di flussi elevati per abbassare i tempi di trattamento, sono i limiti attuali di questa interessante metodica. La produzione on-line di liquido di sostituzione e l'impiego di soluzione di infusione con bicarbonato (per una migliore correzione dell'acidosi) possono rilanciare ed attualizzare questo tipo di tecnica. 


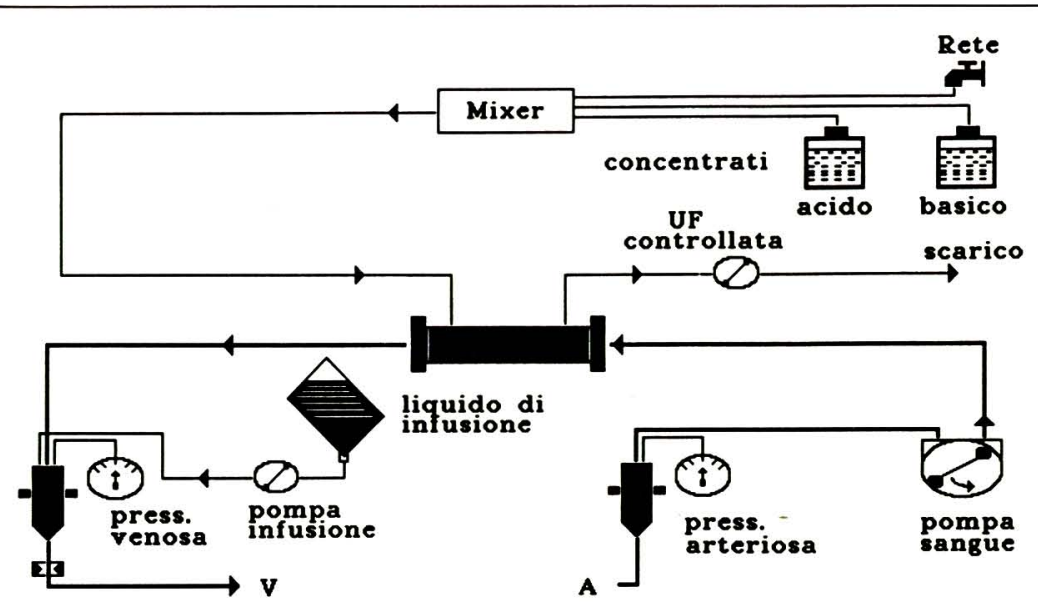

\section{EMODIAFILTRAZIONE}

Membrana

Tampone del bagno dialisi

Flusso bagno dialisi

Flusso sangue

Tampone nel liq. infus.

Infusione totale

Velocità UF

Durata

Apparecchiatura dialitica
Sintetica, ad alta permeabilità diffusiva e convettiva

Bicarbonato (acetato)

$\sim 500 \mathrm{ml} / \mathrm{min}$

$\geq 300 \mathrm{ml} / \mathrm{min}$

Lattato

10-20 L

In base ad infusione e calo ponderale

$<4$ ore

Controllo volumetrico UF, controllo gravimetrico dell'infusione o tramite pompa peristaltica
Fig. 6

\section{Emodiafiltrazione (Fig. 6)}

\section{Vantaggi (Tab. VII)}

La combinazione dei meccanismi di trasporto convettivo e diffusivo può essere la forma più efficiente per la rimozione di un ampio range di soluti e rappresentare una premessa ottimale per eventuali riduzioni del tempo di trattamento.

\section{Svantaggi (Tab. VII)}

Attrezzature dedicate insieme con necessità di elevate quantità di liquido di sostituzione rendono costosa e complessa questa metodica. Il rischio di trasferire dei pirogeni dal dialisato al sangue è minimo ed in ogni caso connesso alla qualità e integrità delle membrane impiegate.

\section{Biofiltrazione senza acetato (AFB) (Fig. 7)}

Vantaggi (Tab. VIII)

I vantaggi sono quelli di una bicarbonato dialisi eseguita con una membrana biocompatibile quale il poliacrilonitrile. La correzione dell'acidosi può essere più facilmente personalizzata ed il rischio di back-filtration come tale non esiste data anche l'improbabile contaminazione del concentrato (ad alta salinità) impiegato.

\section{Svantaggi (Tab. VIII)}

Necessità di attrezzature e soluzioni dedicate. Risulta poco duttile nella quantità di liquidi da scambiare che è strettamente connessa con il bilancio intradialitico dei bicarbonati.
TAB. VII - EMODIAFILTRAZIONE

Vantaggi:

- mebrane biocompatibili

- elevata rimozione di piccole, medie e grandi molecole

- riduzione dei tempi di trattamento

- non effetti destabilizzanti sul sistema cardiovascolare

- maggiori possibilità di personalizzare il bilancio intradialitico di elettroliti e tamponi attraverso semplici modifiche della composizione e velocità di infusione del liquido di sostituzione

\section{Svantaggi:}

- notevole complessità delle apparecchiature dialitiche

- ingenti quantità di liquido di sostituzione e quindi maggiore...

- ...rischio di contaminazione chimica (Al, nitrati, cloramine, elementi traccia) e biologica delle soluzioni infusionali

- alti costi (apparecchiature, soluzioni di infusione)

\section{TAB. VIII - BIOFILTRAZIONE SENZA ACETATO}

Vantaggi:

- eliminazione completa del tampone acetato

- stessi vantaggi di altre tecniche di emodiafiltrazione in termini di biocompatibilità delle membrane ed efficacia depurativa

- possibilità di personalizzare ed ottimizzare la somministrazione (diretta, in via infusiva) di bicarbonato, anche secondo l'applicazione di modelli teorici e quindi...

- ...migliore correzione dell'acidosi

- migliore stabilità cardiovascolare

- assenza delle problematiche tecniche proprie delle apparecchiature che utilizzano bagni dialisi con bicarbonato

\section{Svantaggi:}

- necessità di apparecchiature dedicate

- rischio di bilanci sodici "errati" se si utilizzano inappropriate concentrazioni di sodio nel bagno di dialisi e/o nel liquido di reinfusione

- rigidità nelle quantità di liquido di reinfusione, strettamente connesse al bilancio dei bicarbonati 


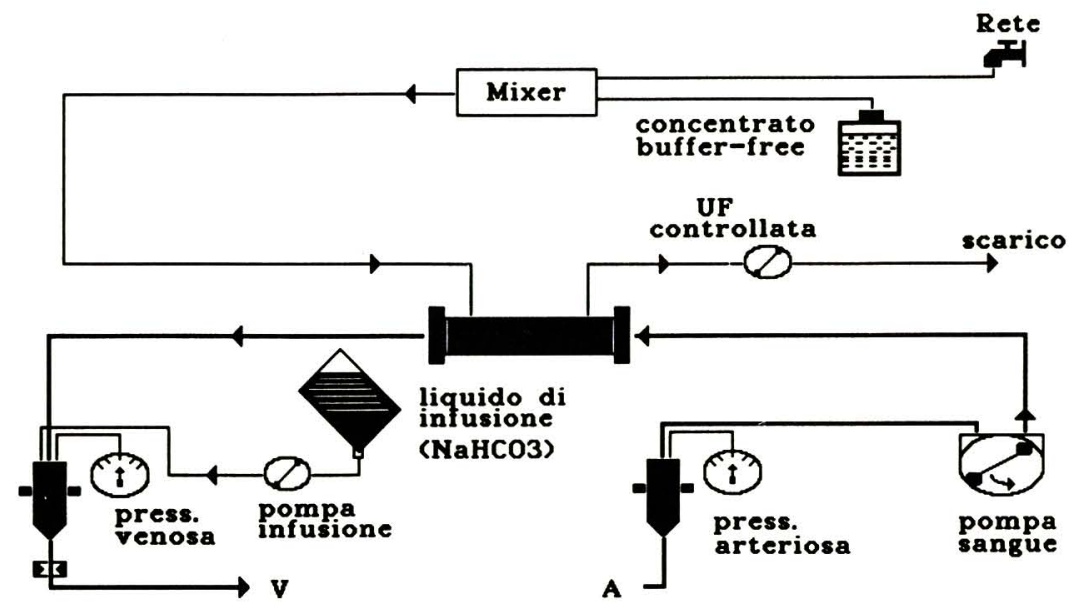

BIOFILTRAZIONE SENZA ACETATO [AFB]

\section{Membrana}

Tampone del bagno dialisi

Flusso bagno dialisi

Flusso sangue

Tampone nel liq. infus.

Infusione totale

Velocità di UF

Durata

Apparecchiatura dialitica
Sintetica, ad alta permeabilità diffusiva e convettiva (PAN)

Assente

$500 \mathrm{ml} / \mathrm{min}$

$>300 \mathrm{ml} / \mathrm{min}$

Bicarbonato

6- 10 litri

In base all'infusione e calo ponderale

$<4$ ore

Controllo volumetrico UF, controllo

dell'infusione tramite pompa peristaltica

Fig. 7

\section{Paired filtration dialysis (PFD) (Fig. 8)}

Vantaggi (Tab. IX)

La separazione fisica e spaziale dei processi convettivi e diffusivi rende più agevole e soprattutto costante in termini di clearance la rimozione dei soluti di differente peso molecolare.

La disponibilità on-line di ultrafiltrato pressoché simile all'acqua plasmatica è una premessa indispensabile per l'ottimizzazione ed individualizzazione della singola seduta emodialitica. E questo è ormai l'obiettivo principe della terapia dialitica degli anni '90.

\section{Svantaggi (Tab. IX)}

L'utilizzo dell'ultrafiltrato nel monitoraggio biochimico della seduta emodialitica presuppone lo sviluppo di biosen- sori dedicati. La gestione di un dializzatore a doppia camera richiede particolari accorgimenti tecnologici e talora espone ad un maggior rischio di coagulazione del doppio filtro.

\section{Scelta ed \\ individualizzazione del trattamento emodialitico}

La scelta di una tecnica rispetto ad un'altra nell'applicazione della terapia dialitica non può limitarsi ad un semplice confronto tra una serie di vantaggi e svantaggi. A parte le considerazioni di carattere teorico che possono essere fatte per l'una o l'altra metodica, sono $i$ costi, la disponibilità di personale e di spazi, le opportunità tecniche e di stoccaggio del materiale, gli elementi premi- nenti che si confrontano con le esigenze cliniche del paziente da trattare. Un giudizio aprioristico non può che essere di parte e quindi non esportabile nelle singole realtà. Molto più realistico ci sembra suggerire una serie di ingredienti (Tab. X) che, commisurati alle disponibilità e variamente combinati possono portare ad una ottimizzazione del trattamento emodialitico sulla base delle caratteristiche del singolo paziente.

L'individualizzazione della terapia dialitica presuppone però sia la scelta del trattamento più idoneo a quel paziente, che l'adattamento del trattamento stesso alle necessità del paziente nella singola seduta dialitica.

L'ampliarsi delle problematiche cliniche della popolazione dei pazienti in dialisi cronica impone una modifica dei nostri atteggiamenti e delle modalità di conduzione della seduta emodialitica. Attualmente aprioristici ed empirici risultano non solo la scelta del tipo di trattamento ma anche i tempi di trattamento, i parametri macchina (flussi, pressioni, temperatura) e la stessa entità del calo ponderale del paziente. Durante la seduta di dialisi ci si limita ad un controllo programmato del peso corporeo e talora della pressione arteriosa. Si interviene con tempestività solo all'occorrenza di complicanze (ipotensione, aritmie) che possono essere fatali per il paziente. Il paziente critico (anziano, vasculopatico) non ha però degli ampi ranges fisiologici ed i limiti tra uno stato di benessere e la comparsa di pericolose complicanze sono estremamente vicini. La scelta dello stesso "peso secco" diventa sempre più critica, e pochi etti di peso corporeo in difetto o in eccesso separano la condizione di equilibrio emodinamico da quella di scompenso. Nello stesso paziente possono alternarsi periodi di dialisi caratterizzate da numerosi episodi collassiali, e pericolosi edemi polmonari acuti nei periodi di interdialisi.

La continua modifica delle variabili indipendenti del trattamento (filtro, composizione del dialisato, flussi, tempi di trattamento, ecc.) accanto ad un monitoraggio continuo delle variabili fisiologiche del paziente (pressione, volemia, ritmo cardiaco, ecc.), stanno diventando elementi indispensabili per un trattamento ottimale.

Gli studi cinetici sono stati sotto questo aspetto il primo esempio di razionalizza- 


\section{TAB. IX - PAIRED FILTRATION} DIALYSIS (PFD)

\section{Vantaggi:}

- ottimizzazione dei fenomeni di trasporto di soluti (per la separazione di convezione e diffusione)

- possibilità di utilizzo di diversi tamponi in associazione

- disponibilità di ultrafiltrato per analisi delle variazioni delle concentrazioni ematiche di soluti ed elettroliti

- minimi rischi di back-filtration

\section{Svantaggi:}

- necessità di apparecchiature dedicate

- limiti attuali di biocompatibilità della membrana del $2^{\circ}$ dializzatore

- necessità di una più accorta anticoagulazione per una maggiore facilità verso fenomeni emocoagulativi a livello della camera di passaggio tra i due filtri

- costi elevati

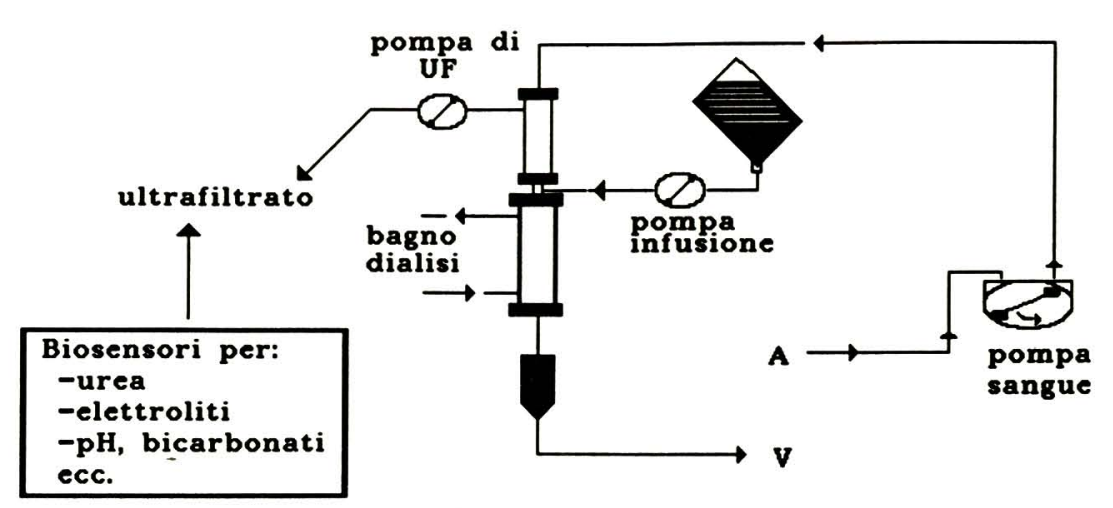

\section{PAIRED FILTRATION DIALYSIS [PFD]}

Membrana

Tampone bagno dialisi

Flusso bagno dialisi

Flusso ematico

Tampone liq. inf.

Infusione totale

Velocità UF

Durata

Apparecchiatura dialitica
Dializzatore a bassa permeabilità emofiltro in polisulfone

Acetato o bicarbonato

$500 \mathrm{ml} / \mathrm{min}$

$300-350 \mathrm{ml} / \mathrm{min}$

Lattato o bicarbonato

Variabile (9-12 L)

Variabile $(<60 \mathrm{ml} / \mathrm{min})$

$<4$ ore

Controllo volumetrico e flussimetrico dell'UF, controllo dell'infusione gravimetrico o con sistema Coriolis

\section{Fig. 8}

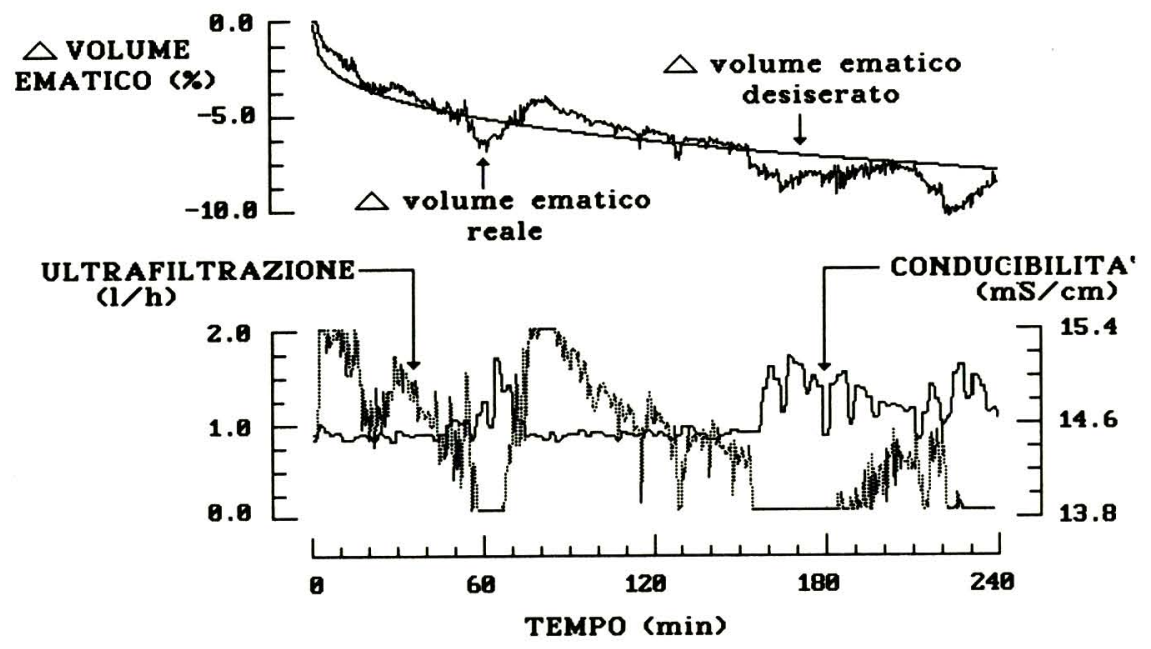

Fig. 9 - Esempio di controllo della volemia in corso di una seduta emodialitica con variazioni in automatico della conducibilità del bagno di dialisi e della velocità di ultrafiltrazione.
TAB. X - INGREDIENTI PER OTTIMIZZARE IL TRATTAMENTO EMODIALITICO

- Uso del bicarbonato come tampone

- Controllo volumetrico dell'ultrafiltrazione e dell'infusione

- Liquido di dialisi sterile

- Biocompatibilità dei materiali costituenti il circuito extracorporeo

- Monitoraggio e modeling dei bilanci di urea, elettroliti e tamponi

- Controllo "on-line" di parametri emodinamici (pressione arteriosa, frequenza cardiaca, volemia, ecc.) e fisici (temperatura corporea, ecc.) 
zione della terapia dialitica. Pur riconoscendo però alla cinetica dell'urea, e soprattutto a Gotch che ne è stato il paladino (11), il merito di aver prospettato la possibilità di quantificare la dose di dialisi adattandola al singolo paziente, non si può limitare l'adeguatezza dialitica all'urea. Soprattutto non si può ritenere l'urea come l'unico indicatore dello stato di intossicazione ed estrapolare dai suoi livelli ematici lo stato di benessere del paziente.

Gli Americani, che hanno sposato e seguito pedissequamente e talora con eccessivo entusiasmo le conclusioni del National Cooperative Dialysis Study (12) sull'adeguatezza dialitica (livelli medi di urea sul tempo - TAC urea inferiori ai $70 \mathrm{mg} / \mathrm{dl}$, e $\mathrm{Kt} / \mathrm{V}=1$ ), si stanno ora interrogando sugli alti tassi di mortalità annua, vicini al $30 \%$, che affliggono la loro popolazione in dialisi (13).

La cinetica dell'urea non può essere vista solo dal lato depurativo ma deve essere associata ad una nutrizione ottimale del paziente, e sopratutto a studi cinetici di elettroliti, quali il sodio, il potassio, il bicarbonato che, forse più dell'urea, condizionano lo stato di benessere del paziente.

Lo studio cinetico, inoltre, non dovrebbe essere visto in senso statico e prospettico, ma adattato alla singola seduta dialitica. Occorre infatti tenere conto della estrema variabilità intraindividuale, da seduta a seduta, dei bilanci idrici ed elettrolitici e del differente accumulo e profilo plasmatico di metaboliti e sostanze "tossiche". D'altronde, se il rene artificiale deve mimare il rene umano, che corregge qualsiasi minima variazione del mezzo interno, non può permettersi delle regolazioni grossolane. Vanno sicuramente affinati i sistemi di informazione e quindi i sensori in grado di fornire un monitoraggio continuo, che è alla base di controlli dinamici e di feed-back automatici. E tutto questo vale non solo sul piano strettamente biochimico ma anche per le grandezze e le variabili fisiologiche e fisiche come la pressione arteriosa e la frequenza cardiaca.

Il monitoraggio e la regolazione in automatico della volemia, che sino ad alcuni anni or sono sembravano solo discorsi futuristici, sono stati da noi applicati nella pratica clinica (14) e stanno diventando una realtà routinaria (Fig. 9).
Sicuramente il prossimo futuro ci riserva la scoperta, la realizzazione e l'applicazione di diversi sensori e biosensori che, impiegati con tecniche adeguate, non solo miglioreranno il nostro livello conoscitivo, ma ci permetteranno di personalizzare le condotte terapeutiche razionalizzando i singoli trattamenti.

Resterà ai Nefrologi il duro compito di confrontare i numerosi vantaggi con gli inevitabili alti costi delle più recenti innovazioni tecnologiche.

\section{BIBLIOGRAFIA}

Kolff W. New ways of treating uremia. London: JA Churchill 1946.

Geerlings W, Tufveson G, Brunner FP, et al. Combined report on regular dialysis and transplantation in Europe, 1990. Nephrol Dial Transplant 1991; 6: 529.

3 Fassbinder W, Brunner FP, Brynger $\mathrm{H}$, et al. Combined report on regular dialysis and transplantation in Europe, 1989. Nephrol Dial Transplant 1991; 6: 535.

4 Ahmad S, Blagg CR, Scribner BH. Center and home chronic hemodialysis. In: Schrier RW, Gottschack CW, eds. Disease of the kidney, ed. 5. Boston: Little Brown, 1992.

Santoro A, Spongano M, Zucchelli P. Il paziente "a rischio" di instabilità cardiovascolare intradialitica. In: Minetti L, Orlandini GC, Petrella E, eds. L'emodiafiltrazione ed i trattamenti ad alti flussi. Milano: Wichtig Editore, 1989.

Dinarello CA. Interleukin-1 and Tumor Necrosis Factor and their naturally occurring antagonist during hemodialysis. Kidney Int 1992; 42: 368-377.
Ronco C. Backfiltration in clinical dialysis: nature of the phenomenon, mechanisms and possible solutions. Int $\mathbf{J}$ Artif Organs 1990; 13: 11-21.

Ebben J, Hirsch O, Lughmann G, et al. Microbiological contaminations of liquid bicarbonate concentrate (LBC) for hemodialysis (HD). ASAIO 1987; 16: 43.

Buoncristiani U, Ragaiolo $M$, Petrucci V, et al. Biofiltration with buffer-free dialysate. Int $\mathbf{J}$ Artif Organs 1986; 9: 9-14.

Ghezzi PM, Frigato G, Fantini GF, et al. Theoretical model and first clinical results of the paired filtration-dialysis (PFD). Life Support Systems 1983; 1 (S1): S271-4.

11 Gotch FA, Sargent JA, Keen ML, et al. Individualized, quantified dialysis therapy of uremia. Proc Clin Dial Transplant Forum 1974; 4: 27.

12 Lowrie EG, Laird NM, eds. Cooperative Dialysis Study. Kidney Int 1983; 23 (S13): 1-122.

13 Hull RA, Parker Tom F. III: Introduction and summary. Proceedings from the Morbidity, Mortality and Prescription of Dialysis Symposium. Am J Kidney Dis 1990; 15: 375-83.

14 Santoro A, Mancini E, Spongano M, et al. Sistemi di monitoraggio emodinamico e di controllo automatico nella conduzione della seduta emodialitica. Aggiornamenti Nefrologici della Magna Grecia, Atti del VI corso: $1991 ; 49-62$. 